\title{
THE
}

\section{A graphene-based hydrogel monolith with tailored surface chemistry for PFAS passive sampling}

Jitka Becanova

University of Rhode Island

Zachary S.S.L. Saleeba

Aidan Stone

Anna R. Robuck

University of Rhode Island

Robert H. Hurt

Follow this and additional works at: https://digitalcommons.uri.edu/gsofacpubs

The University of Rhode Island Faculty have made this article openly available.

Please let us know how Open Access to this research benefits you.

This is a pre-publication author manuscript of the final, published article.

Terms of Use

This article is made available under the terms and conditions applicable towards Open Access

Policy Articles, as set forth in our Terms of Use.

\section{Citation/Publisher Attribution}

Becanova, J., Saleeba, Z. S.S.L., Stone, A., Robuck, A. R., Hurt, R. H., \& Lohmann, R. (2021). A graphenebased hydrogel monolith with tailored surface chemistry for PFAS passive sampling. Environmental Science: Nano. Advance Article. https://doi.org/10.1039/D1EN00517K Available at: https://doi.org/10.1039/D1EN00517K

This Article is brought to you for free and open access by the Graduate School of Oceanography at DigitalCommons@URI. It has been accepted for inclusion in Graduate School of Oceanography Faculty Publications by an authorized administrator of DigitalCommons@URI. For more information, please contact digitalcommons-group@uri.edu. 


\section{Authors}

Jitka Becanova, Zachary S.S.L. Saleeba, Aidan Stone, Anna R. Robuck, Robert H. Hurt, and Rainer Lohmann

This article is available at DigitalCommons@URI: https://digitalcommons.uri.edu/gsofacpubs/814 


\section{A graphene-based hydrogel monolith with tailored surface chemistry for PFAS passive sampling}

Jitka Becanova ${ }^{* \#}$, Zachary S.S.L. Saleeba ${ }^{2 *}$, Aidan Stone ${ }^{2}$, Anna R. Robuck ${ }^{1}$, Robert H. Hurt"\#, Rainer Lohmann ${ }^{1}$

${ }^{1}$ Graduate School of Oceanography, University of Rhode Island, Narragansett, RI, United States

${ }^{2}$ School of Engineering, Brown University, Providence, Rl, United States

* authors contributed equally

\# corresponding authors (becanova@uri.edu, robert hurt@brown.edu)

\section{Table of contents}

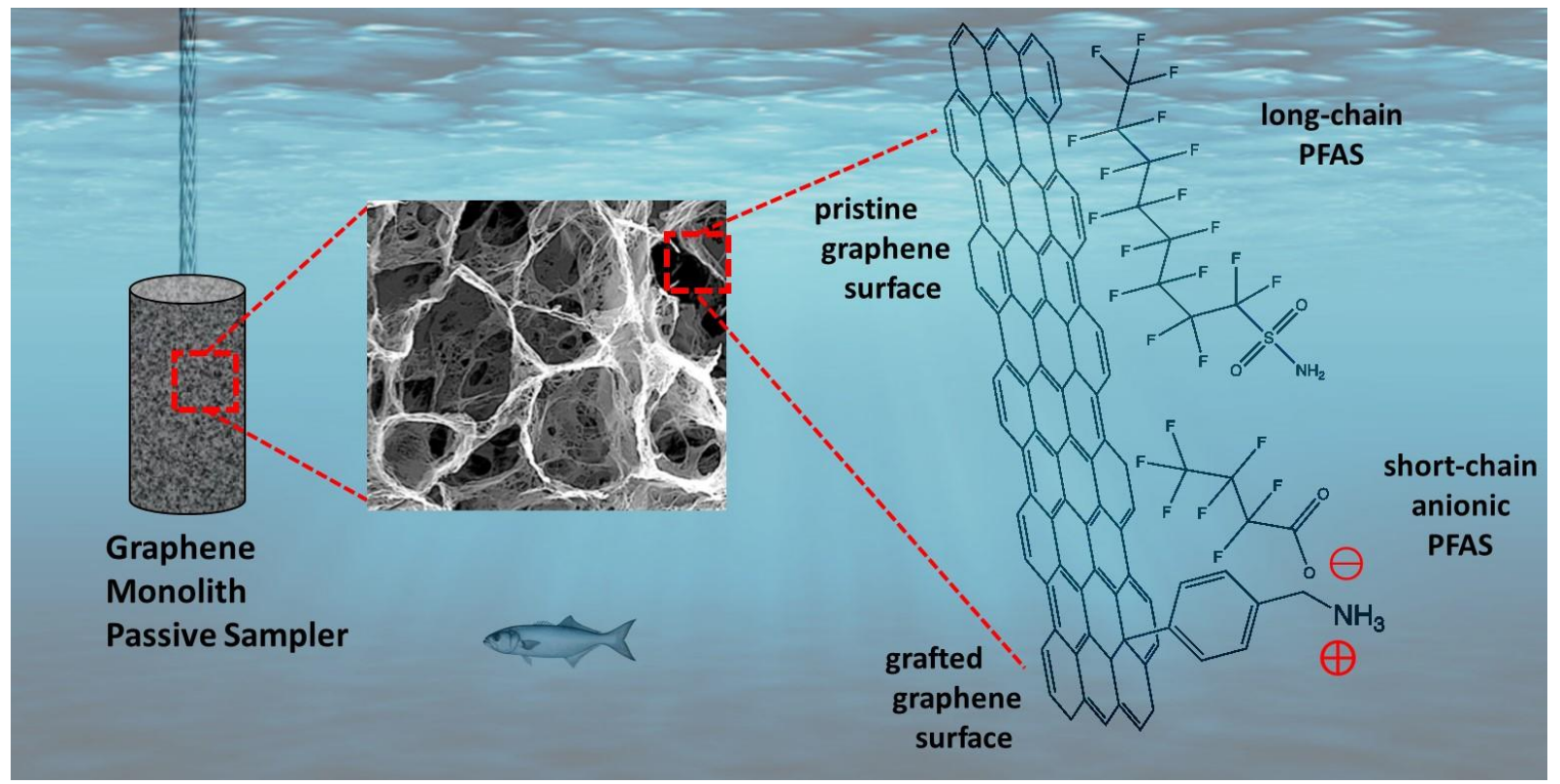

We introduce new PFAS passive sampling devices based on porous graphene monoliths grafted with cationic functional groups for broad-spectrum PFAS pre-concentration including short-chain anionic species.

\section{Environmental Significance Statement}

The aquatic environment is a key medium that mediates human exposure to various pollutants via drinking water and consumption of aquatic food with bioaccumulated contaminants. Per- and polyfluorinated alkyl substances (PFAS) are among the most important aqueous pollutants and are toxic, bioaccumulative, degradation resistant, and globally distributed. The scientific community is still lacking effective and sensitive tools for their environmental monitoring, especially when they are present in low concentrations. In this study a graphene-based porous 
monolith was designed, fabricated, surface functionalized and characterized as a passive sampler for PFAS in water. One formulation of the graphene monolith sampler shows great potential for equilibrium sampling and pre-concentration of broad range of PFAS as a promising tool for monitoring of emerging organic contaminants in aquatic environments.

\begin{abstract}
Aquatic contamination by per- and polyfluorinated alkyl substances (PFAS) has attracted global attention due to their environmental and health concerns. Current health advisories and surface water regulatory limits require PFAS detection in the parts per trillion (ppt) range. One way to achieve those low detection limits is to use a reliable passive sampling-based monitoring tool for PFAS, as exists for numerous nonpolar persistent organic pollutants. Here we introduce a new graphene-based hydrogel monolith and describe its synthesis, chemical functionalization, property characterization, and testing as a PFAS equilibrium passive sampler. The graphene monoliths were self-assembled by hydrothermal treatment from graphene oxide (GO) aqueous dispersions to produce free standing cylinders of $\sim 563 \mathrm{~mm}^{3}$ volume consisting of $\sim 4 \mathrm{wt}-\%$ thinwalled porous graphene and $\sim 96$ wt-\% water. The uptake of 23 PFAS was measured on the asproduced monoliths, and equilibrium partition coefficients $\left(\mathrm{K}_{\mathrm{sw}}\right)$, were derived for longer chain $(C \geq 8)$ perfluoroalkyl acids (PFAA) and neutral precursors such as sulfonamides (log $K_{\text {sw }}$ range 1.9 - 3.6). To increase the $K_{s w}$ for shorter chain PFAA, the monoliths were chemically modified by a new diazonium-based grafting reaction that introduces positive surface charge without damage to the graphenic backbone. Introduction of benzylamine moieties through the diazonium intermediate switches zeta potential at $\mathrm{pH} 7$ from $-45 \mathrm{mV}$ (as-produced graphene) to $+5 \mathrm{mV}$. This modification increased the sorption of short and middle chain PFAA by ten-fold (e.g. log $\mathrm{K}_{\mathrm{sw}}$ for PFBA increased from 1.3 to 2.2), thereby improving the functionality of the passive sampler device for a wider range of PFAS. Field deployments demonstrated that the graphene monoliths were capable of detecting key PFAS in the Delaware River.
\end{abstract}

\title{
Introduction
}

Per- and polyfluorinated alkyl substances (PFAS) are manmade chemicals extremely resistant to environmental degradation. Due to their unique physico-chemical characteristics (e.g. ability to lower the interfacial or/and surface tension, conferring water, stain and oil repellency), PFAS are used in many industrial applications (e.g. polymer and photographic industries, photolithography and manufacturing of semi-conductors) ${ }^{1}$ and are components of various raw materials as well as common consumer products ${ }^{2}$. Many applications directly release PFAS into all environmental 
compartments ${ }^{3-5}$ and pose a potential risk of contamination to a drinking water source ${ }^{6}$ or contribute to secondary PFAS sources such as wastewater treatment plant effluents ${ }^{7,8}$ or landfill leachates ${ }^{9-11}$.

PFAS are known as immunotoxic environmental contaminants ${ }^{12-14}$ and were systematically reviewed by the International Agency for Research on Cancer (IARC) as chemicals with carcinogenic potential. In 2016 perfluorooctanoic acid (PFOA) was classified as a group 2B chemical, a possible human carcinogen ${ }^{15}$. Additionally, the U.S. Environmental Protection Agency (EPA) classified two other PFAS, perfluorooctanesulfonate (PFOS) and hexafluoropropylene oxide-dimer acid HFPO-DA, as having suggestive evidence of carcinogenic potential ${ }^{16-18}$. Due to the combination of PFAS persistence, their toxic potential and worldwide dispersion, PFOS and PFOA have been listed under the Stockholm convention (SC) of persistent organic pollutants ${ }^{19,20}$ and the global production and application of these PFAS is banned or restricted.

The release of PFAS to the aquatic environment has caused extensive background exposure to PFAS that affect the global population via drinking water ${ }^{21}$. In 2017 the U.S. EPA issued drinking water health advisories for two common PFAS: PFOA and PFOS at $70 \mathrm{ng} / \mathrm{L}$ for combined concentrations. More recently, individual US states and countries all around the world have issued more stringent drinking water guidelines for either the sum of multiple PFAS, or the individual congeners ${ }^{22,23}$. Increasing regulatory attention highlights both the continuing concern over human exposure to PFAS and the need for an ability to measure time weighted average (TWA) concentrations, and to better assess PFAS sources, transport and exposure.

Passive samplers are a robust tool for deriving the TWA concentration of pollutants in water, but there is a lack of suitable options for PFAS. Most efforts on passive sampling for PFAS have focused on validating the Polar Organic Chemical Integrative Sampler (POCIS) sampler developed by the United States Geological Survey ${ }^{24-29}$. The drawbacks of the POCIS include the dependency of PFAS uptake rates on water flow velocity caused by the changeable water boundary layer (WBL) on the membrane surface ${ }^{30}$. As an alternative, passive samplers based on diffusive gradients in thin hydrogel films (DGT) were recently modified for PFAS sampling in water ${ }^{31,32}$. However, the agarose-based DGT sampler has low long-term stability in surface water and limited capacity for PFAS beyond PFAA ${ }^{32}$.

Here we pursue a new approach for PFAS passive sampling, based on the self-assembly of graphene oxide nanosheets into porous "monoliths" (non-particulate-based, one-piece, connected, free-standing objects) of controlled shape. The 2004 discovery of graphene ${ }^{33}$ has led 
to a family of mass-produced sheet-like carbon materials that are now available as enabling components in environmental protection and remediation technologies ${ }^{34-36}$. In these applications, collections of graphene nanosheets are assembled into powders, films, or three-dimensional bodies with high internal surface area and surface chemistries tailored for the target contaminants. Graphene has exceptional properties but is not competitive in some large-scale applications where lower-performing, but lower-cost commodity porous carbons can also be applied. In contrast, miniature passive sampling devices are an ideal application for graphene materials due to the small amounts of base material required (milligrams per device) coupled with the stringent performance requirements, which include high contaminant uptake capacity, purity, monolithic form, and high contaminant recovery by extraction.

This article describes the fabrication of shape-controlled graphene hydrogel monoliths, their physical properties and PFAS adsorption / extraction performance of relevance to equilibrium passive sampling devices, followed by a field deployment to test its performance under realistic conditions. A new method of surface modification by diazonium covalent grafting is also demonstrated, which increases the partition coefficients for short chain PFAA compounds and broadens the spectrum of PFAS that can be effectively sampled and detected.

\section{Methods}

Graphene monolith synthesis

Graphene Oxide (GO) nanosheet suspensions were prepared by a modified Hummers' method described in detail by Liu et.al ${ }^{37}$, yielding a solid product with $\mathrm{C}: \mathrm{O}$ atomic ratio of $\sim 2.0$ and trace amounts of $\mathrm{S}, \mathrm{N}$ and $\mathrm{Cl}$ impurities $(<0.52 \%)$ determined by $\mathrm{x}$-ray photoelectron spectroscopy (XPS). $10 \mathrm{~mL}$ of GO nanosheet suspensions at a concentration of $2 \mathrm{mg} \cdot \mathrm{mL}^{-1}$ were loaded into a $15 \mathrm{~mL}$ Teflon lined stainless steel autoclave and heated to $180^{\circ} \mathrm{C}$ for $10 \mathrm{hrs}$. After several hours of cooling, a self-assembled cylindrical hydrogel monolith was removed, and its surface blotted dry for weighing, characterization, and use.

\section{Chemical functionalization}

The graphene monolith (GM) in its as-produced form carries a negative surface charge due to deprotonation of acidic oxygen-containing functional groups that are a common feature on asproduced carbon surfaces (see Results). This negative surface charge introduces a repulsive electrostatic force component for anionic PFAS, and in particular may suppress adsorption of short-chain varieties where the attractive hydrophobic forces are less pronounced. Introducing positively charged functional groups can reduce or even flip the sign of the net surface charge, 
and we hypothesize should increase affinity for short-chain PFAA. Here we covalently grafted 4aminobenzylamine (4-ABA) to the graphene surfaces by adapting a chemical pathway based on diazonium intermediates that has been demonstrated previously on carbons for different arylamine precursors ${ }^{38-40}$. The procedure exposes the graphene monolith to a slightly acidic solution with $0.15 \mathrm{M}$ hydrochloric acid (37\%, Fisher Scientific, MA, USA), $0.085 \mathrm{M}$ 4-ABA (98\%, Acros Organics, NJ, USA), and $0.085 \mathrm{M}$ sodium nitrite (99\%, Fisher Scientific, MA, USA) at $80^{\circ} \mathrm{C}$ for a tested range of 2 to $18 \mathrm{hrs}$ with gentle stirring on a shaker table. Sodium nitrite is added last, after the monolith is placed in the solution. The reaction takes place in $50 \mathrm{~mL}$ round bottom flasks submerged in mineral oil on a heated magnetic stir plate. The graphene monolith is then washed in methanol (Fisher Scientific, MA, USA), tetrahydrofuran (99.9\%, Mallinckrodt Chemicals, NJ, USA) and DI water sequentially for $24 \mathrm{hrs}$ each.

\section{Monolith property characterization}

Thermal gravimetric analysis (TGA) was performed on a Mettler Toledo TGA/DSC 1 Star to determine water content and confirm GO reduction during the hydrothermal treatment. TGA tests were run on $10 \mathrm{mg}$ samples in $150 \mu \mathrm{L}$ alumina crucibles under a nitrogen flow of $80 \mathrm{~mL}$. $\mathrm{min}^{-1}$ with a temperature ramp rate of $10 \mathrm{~mL} \cdot \mathrm{min}^{-1}$ from $25^{\circ}$ to $800^{\circ} \mathrm{C}$. All scanning electron microscopy (SEM) images (LEO 1530) were acquired at $20 \mathrm{kV}$. X-ray diffraction (XRD) analyses wereas performed on a Bruker D8 Discovery 2D X-ray Diffractometer mounted on to a Si substrate in which all crystalline peaks from substrate were removed. X-ray photoelectron spectroscopy (XPS) work was performed on a Thermo Fisher K-Alpha X-ray Photoelectron Spectrometer. SEM and XPS samples were freeze-dried prior to imaging.

Surface charge was characterized by measuring zeta potential of both as-produced and functionalized graphene monoliths as a function of solution $\mathrm{pH}$. The monolith was sectioned into $1 \mathrm{~mm} \times 1 \mathrm{~mm}$ pieces and then dispersed in $15 \mathrm{~mL}$ of DI water with $0.1 \mathrm{mM} \mathrm{NaCl}$ (Fisher Scientific, MA, USA) as background electrolyte and sonicated for 20 minutes to create small debris particles in suspension whose surface (zeta) potentials could be determined by electrophoretic measurements. $\mathrm{pH}$ was controlled by titration with small amounts of $\mathrm{HCl}$ or $\mathrm{NaOH}$. Zeta potentials were then measured at 25 $\mathrm{C}$ using a Malvern Nano ZS.

Methylene blue (MB) dye was used as a model adsorbate to estimate the graphene monolith surface area available to aqueous-phase organic contaminants. Initial concentrations of the cationic dye, MB (Aldrich Chemical Company, St. Louis, MO), ranged from 4 to $170 \mathrm{mg} \cdot \mathrm{L}^{-1}$ in a $100 \mathrm{~mL}$ solution. A single intact graphene monolith was added to the $100 \mathrm{~mL}$ solutions with varying MB concentration and shaken at $100 \mathrm{rpm}$ on a shaker table continuously while samples 
were taken at various time points to determine an equilibrium concentration of $\mathrm{MB}$. These concentrations were measured by UV-Vis spectroscopy (Jasco V-730) at a fixed wavelength of $664 \mathrm{~nm}$ with some requiring dilution to be within the appropriate concentration range of the spectrometer. Spectral measurements were also performed to verify that there was no peak shift due to dimer formation at higher concentrations. Initial calibrations were made in the range of diluted samples, and daily single point calibrations were performed thereafter. The dry solid mass of a single monolith device used for normalizing adsorbate uptake values was determined gravimetrically after oven drying at $65^{\circ} \mathrm{C}$ for $24 \mathrm{hrs}$.

\section{PFAS standards}

The solid PFAS standards for laboratory experiments: perfluoro-n-butanoic acid (PFBA); perfluoro-n-pentanoic acid (PFPeA); perfluoro-n-hexanoic acid (PFHXA); perfluoro-n-heptanoic acid (PFHpA); perfluoro-n-octanoic acid (PFOA); perfluoro-n-nonanoic acid (PFNA); perfluoro-ndecanoic acid (PFDA); perfluoro-n-undecanoic acid (PFUnDA); perfluoro-n-dodecanoic acid PFDoDA); perfluoro-n-tridecanoic acid (PFTrDA); perfluoro-n-tetradecanoic acid (PFTeDA); perfluoro-1-butanesulfonate (PFBS); perfluoro-1-hexanesulfonate (PFHXS); perfluoro-1-heptanesulfonate (PFHpS); perfluoro-1-octanesulfonate (PFOS); perfluoro-1-butanesulfonamide (FBSA); perfluoro-1-octanesulfonamide (FOSA); N-methylperfluoro-1-octanesulfonamide (MeFOSA); $\mathrm{N}$ ethylperfluoro-1-octanesulfonamide (EtFOSA); $1 \mathrm{H}, 1 \mathrm{H}, 2 \mathrm{H}, 2 \mathrm{H}$-perfluoro-1-hexane sulfonate (4:2 FTS); $1 \mathrm{H}, 1 \mathrm{H}, 2 \mathrm{H}, 2 \mathrm{H}$-perfluoro-1-octane sulfonate (6:2 FTS); $1 \mathrm{H}, 1 \mathrm{H}, 2 \mathrm{H}, 2 \mathrm{H}$-perfluoro-1-decane sulfonate (8:2 FTS); and 2,3,3,3-tetrafluoro-2-(1,1,2,2,3,3,3-hepta-fluoro-propoxy)-propanoic acid (HFPO-DA) - purity 95-97\%; were purchased from SynQuest Labs (FL, USA), Santa Cruz Biotechnology (TX, USA) and Toronto Research Chemicals (Ontario, Canada). The solutions of individual compounds (concentration approx.10 mg.mL-1) were created by dissolving approximately $30 \mathrm{mg}$ of each compound (exact mass recorded) in $3 \mathrm{~mL}$ of LC/MS grade methanol (Honeywell Riedel-de Haën, Germany). The working solution mixtures (concentration $200 \mu \mathrm{g} \cdot \mathrm{mL}^{-1}$ ) of various PFAS were prepared by mixing individual PFAS in LC/MS grade methanol.

The 8-point calibration curve $\left(0.001-100 \mathrm{ng}_{\mathrm{mL}} \mathrm{m}^{-1}\right), \mathrm{QA} / \mathrm{QC}$ instrumental performance check, and surrogate standard were created using analytical PFAS standards purchased from Wellington Laboratories (Ontario, Canada). Specifically, PFAC-24PAR; MPFAC-24ES; N-MeFOSA-M; NEtFOSA-M; FBSA-I; FHxSA-I; HFPO-DA; d-N-MeFOSA-; d-N-EtFOSA-M; and M3HFPO-DA were used. Individual target PFAS and corresponding isotope labelled analogues are listed in Table SI 1. 


\section{$\underline{\text { LC-MS/MS analysis }}$}

The LC-MS/MS analysis of targeted PFAS in laboratory experiments relied on a liquid chromatograph (Shimadzu Prominence UFLC) equipped with Gemini C18 hybrid column (3 $\mu \mathrm{m}$, $2.1 \mathrm{~mm}$ X $50 \mathrm{~mm}$; Phenomenex) coupled to mass spectrometer (AB Sciex 4500 QTRAP) operating in negative mode. To reduce background contamination in the system, a delay column (Luna $5 \mu \mathrm{m}$ C18(2) $100 \AA$, LC Column $30 \times 2$ mm) was installed to the LC system. For analysis, $20 \mu \mathrm{L}$ of prepared extract was injected on the analytical column and PFAS were separate and determined (all analytical details are listed in SI, Table SI 2, 3 and 4). The LC-MS/MS analysis of targeted PFAS in-field deployment samples was performed using a Vanquish UPLC system (Thermo Fisher Scientific, Waltham, MA) equipped with a heated Accucore C18+ column (2.1 mm x $100 \mathrm{~mm}$ x 1.5 um particles) coupled with a Thermo Orbitrap Fusion mass spectrometer (Thermo Fisher Scientific, Waltham, MA) with a heated electrospray ionization (HESI) source operated in negative mode ${ }^{41}$.

\section{PFAS Partitioning Experiments}

PFAS partitioning experiments were performed in high density polyethylene bottles (Nalgene® Fisher Scientific, MA, USA) to minimize PFAS sorption to secondary surfaces. For each experiment a single graphene monolith was exposed to a PFAS aqueous solution (DI water with pH 5.5 - 5.8 and PFAS concentration between 5 and $50 \mathrm{ng} \cdot \mathrm{mL}^{-1}$ and the experiment repeated in triplicate. To maintain linear flow conditions during the partitioning experiments, the centrifuge tubes were vertically shaken on a platform shaker at a moderate speed (100 rpm). Same experiments were utilized to study: i) elution profiles of PFAS, ii) adsorption isotherms and iii) partition coefficients for graphene monolith in both, pristine and modified form.

At the end of each experiment, the monolith was drained for 30 seconds prior to its transfer to 2 $\mathrm{mL}$ of LC/MS grade methanol in $15 \mathrm{~mL}$ polypropylene centrifuge tube. Time-dependent adsorption experiments were carried out from $1 \mathrm{hr}$ to $48 \mathrm{hrs}$ on 16 PFAS species to establish criteria for reaching equilibrium. Data in Table SI 5; and Figure SI 1 show equilibration times on the order of $12 \mathrm{hrs}$, and $24 \mathrm{hrs}$ was selected as a safe time point for all further experiments. As a next step, the elution profile of solid liquid extraction of monolith was established (see Table SI 6 and Figure SI 2), and two subsequent extraction steps were assessed as sufficient for PFAS extraction and utilized for all partitioning experiments.

Particularly, $2 \times 2 \mathrm{~mL}$ of monolith's extracts were combined from each experiment, spiked with 50

$\mu \mathrm{L}$ of PFAS surrogate standard (final concentration $2 \mathrm{ng} \cdot \mathrm{mL}^{-1}$ ) and evaporated under a gentle stream of nitrogen to the final volume of $500 \mu \mathrm{L}$. Prior the instrumental analysis, the aliquot of 
$80 \mu \mathrm{L}$ of monolith's extract was diluted using $120 \mu \mathrm{L}$ of $4 \mathrm{mM}$ ammonium acetate (LiChropur, Supelco, PA, USA) in LC/MS grade water (Fisher Scientific, MA, USA). Similarly, the $600 \mu \mathrm{L}$ aliquot of PFAS aqueous solution was spiked with $50 \mu \mathrm{L}$ of surrogate standard (final concentration $2 \mathrm{ng} \cdot \mathrm{mL}^{-1}$ ) and mixed with $350 \mu \mathrm{L}$ of $4 \mathrm{mM}$ ammonium acetate in LC/MS grade methanol prior the instrumental analysis.

A sampler/water partition coefficient $\left(\mathrm{K}_{\mathrm{sw}}\right)$ was calculated as:

$$
K_{S W}=\frac{q}{C} \quad E q .1,
$$

where $\mathrm{q}$ is the adsorbed amount (moles adsorbate per mass of adsorbent) and $C$ is equilibrium concentration (moles per mass of solution) making $\mathrm{K}_{\mathrm{sw}}$ dimensionless.

\section{Mass balance experiments}

To calculate the mass balance, the partitioning experiment was performed using 3 graphene monoliths exposed in $500 \mathrm{~mL}$ of aquatic solution of PFAS (10 ng. $\left.\mathrm{mL}^{-1}\right)$ for $24 \mathrm{hrs}$. The mass balance was calculated by combining the total amount of individual PFAS in both phases (graphene monolith extract and water) and comparing it to the mass of the originally introduced PFAS. For most of the compounds the mass balance fell between 59\% and $124 \%$ (median $71 \%$ ) as seen in Table SI 7. The compounds with recoveries lower than 59\% were either PFAAs with very long chains $(\geq \mathrm{C} 12$ ) or sulfonamides (MeFOSA and EtFOSA). The lower recovery for these hydrophobic compounds could be caused by one of two phenomena: $i)$ their high tendency to sorb to all surfaces, including walls of HDPE exposure bottles, caused by their hydrophobic character ${ }^{42}$, and/or ii) their irreversible sorption to the graphene monolith.

\section{Field deployments}

Modified graphene monoliths were deployed in Delaware River (New Jersey, USA) at localities with known high concentration of legacy (e.g., PFOA, PFOS and PFNA) and emerging PFAS ${ }^{41 .}$ Graphene monoliths in triplicates were arranged in stainless-steel cages (Figure SI 7) at 3 locations (Site A, B \& C) for 7 days. Recovered samplers were immediately placed into extraction solution (methanol), spiked with the mass labeled PFAS analogs and prepared for the LC-MS/MS analysis (see sample preparation details above). The results were contrasted to background contamination in field blanks which undergo the same procedure as the deployed samplers and compare to PFAS determined in grab water samples (see SI for details). 


\section{Results and Discussion}

Graphene monolith synthesis and characterization

To create an effective passive sampling device, we attempted to make a single-piece, shaped material (a monolith, not a powder) that preserves as much as possible the high specific surface area of individual atomically-thin graphene nanosheets. Figure 1a shows the selected hydrothermal synthesis route that begins with $10 \mathrm{~mL}$ of $\mathrm{GO}$ stock suspension (2 mg. $\mathrm{mL}^{-1}$ ) processed at $180^{\circ}$ Celsius in a $15 \mathrm{~mL}$ Teflon-lined autoclave. The result is a cylindrical monolith (Fig. 1c) approximately $6.8 \mathrm{~mm}$ in diameter and $15.5 \mathrm{~mm}$ in height. After formation, the excess liquid is optically clear, indicating that almost all of the original nanosheets became associated with the solid monolith during the hydrothermal assembly process. After blotting to remove surface

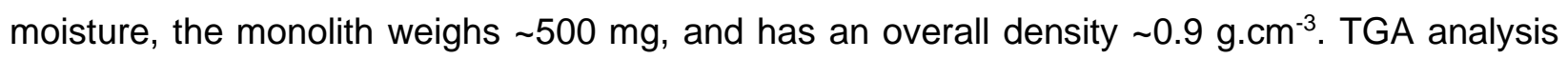
indicates $~ 96$ wt-\% water (Fig. SI 3), which together with the density value indicates that the internal spaces of this as-produced monolith are nearly filled (saturated) with liquid $\mathrm{H}_{2} \mathrm{O}$ as depicted in figure $1 \mathrm{~d}$. The monolith is thus classified as a hydrogel, where the residual hydrophilic oxygen functional groups on the reduced GO (rGO) provide hydrogen bonding sites for retention of water molecules within the complex internal porous structure ${ }^{43}$. After thermal drying for $24 \mathrm{hr}$ at $65^{\circ} \mathrm{C}$, the monolith mass falls dramatically to an average of $11.2 \mathrm{mg}$ with a standard deviation of $0.35 \mathrm{mg}$ and standard error of $3 \%$ based on 5 samplers chosen from two synthesis batches fabricated at different times. The dry mass allows us to estimate the graphene product yield, which is $11.2 \mathrm{mg} / 20 \mathrm{mg}$-starting-GO or $56 \%$, which is a value similar to the typical mass loss associated with the conversion of GO to reduced GO (rGO) by thermal reduction methods. This dry solids' mass $(11.2 \mathrm{mg}$ ) is used later to normalize adsorbed amounts of PFAS for adsorption isotherms.

Figure $1 \mathrm{~b}$ shows that the process of random nanosheet coalescence creates a cellular-foam-like internal structure consisting of thin walls surrounding cavernous pores that range in size from 2

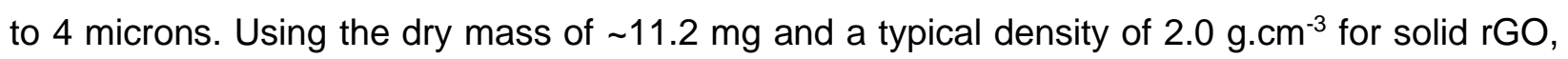
the as-produced product has a solid volume fraction of only $1 \%$. We hypothesize that this extremely large pore volume (99\%) when saturated with water in the immersed stated will allow for rapid equilibration during sampling, especially relative to other sampling technologies that require PFAS solid state diffusion through non-porous polymeric material. This hypothesis is tested using time-dependent adsorption experiments (described later) and the results used to establish criteria for reaching the equilibrium regime. Figure SI 4 is a SEM image that shows a skin-like outer layer, which is still porous but of higher solid density than the core. This skin layer appears to aid in the mechanical stability of the monolith against handling and processing 
stresses. Interestingly, during elevated temperature drying $\left(65^{\circ} \mathrm{C}\right)$, the monolith shrinks irreversibly by a factor of $\sim 4$ in each linear dimension to $\sim 1 / 64^{\text {th }}$ of its original hydrated volume. This highlights the importance of water in forming and maintaining the hydrogel structure.

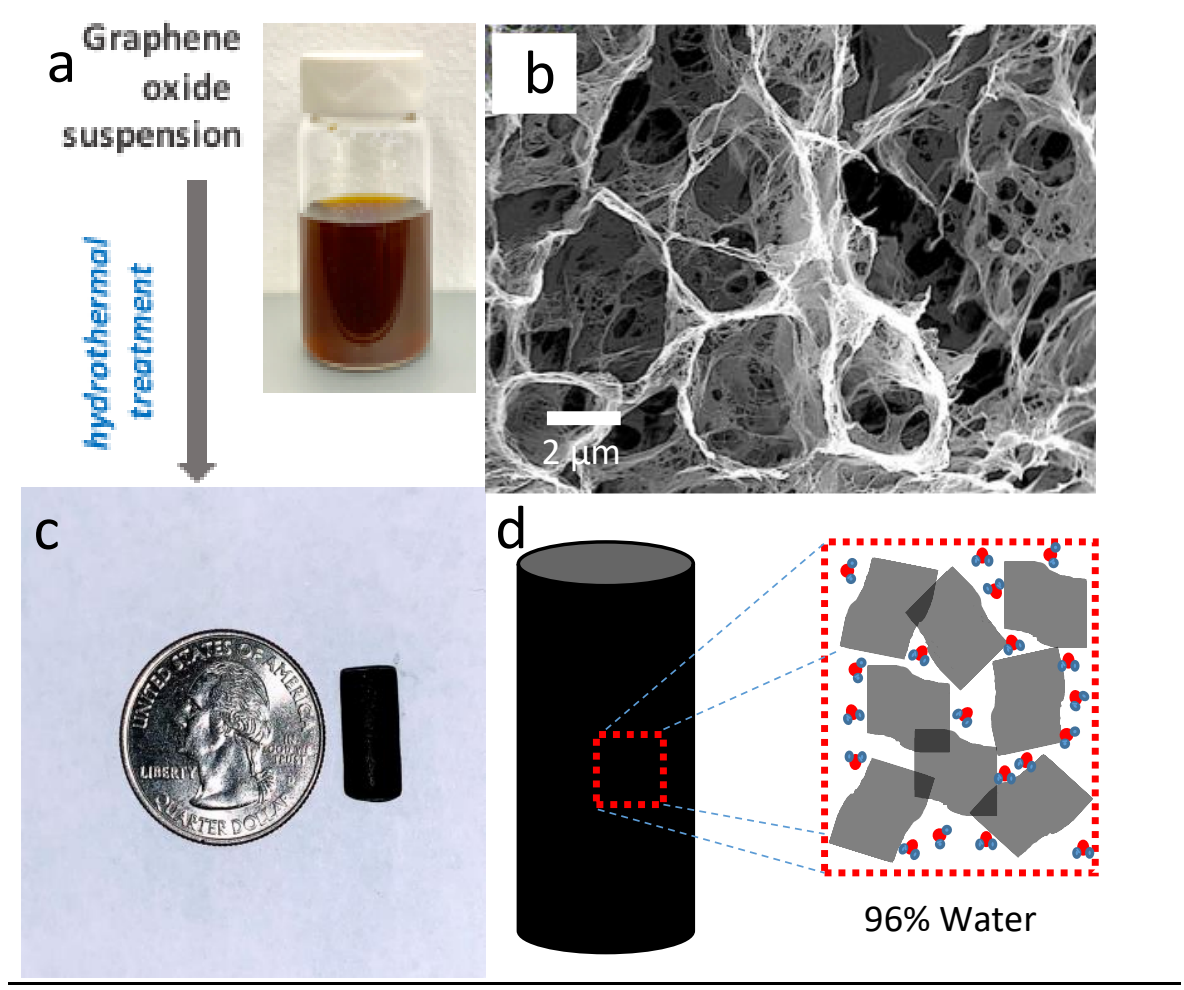

Figure 1: Synthesis and structure of the graphene hydrogel monolith. (a) Graphene oxide nanosheet suspension synthesized by a modified Hummers' method $(2 \mathrm{mg} / \mathrm{ml}$ stock suspension). (b) SEM image showing a foam-like structure in the interior of the monolith, with micron-sized pores surrounded by thin graphene-based walls. (c) Hydrothermal treatment leads to the porous graphene monolith product shown with scale reference. (d) Sketch showing the hydrophobic sheet-to-sheet interactions leading to gelation and the water association on the remaining oxygen-containing surfaces.

Figure $2 \mathrm{a}$ and $\mathrm{b}$ show a methylene blue $(\mathrm{MB})$ adsorption isotherm at $20^{\circ} \mathrm{C}$, used to estimate the total surface area of the monolith when immersed in aqueous media, as in the intended passive sampling application. The monolith has a maximum capacity approaching $300 \mathrm{mg}-\mathrm{MB} \cdot \mathrm{g}^{-1}-\mathrm{rGO}$. For comparison, literature studies on suspended precursor GO nanosheets (which are isolated single-layer materials) reported capacities from 671 to $1450 \mathrm{mg} \cdot \mathrm{g}^{-1} 44$ which suggests that the monolith internal cavity walls consist of only a few graphene layers. The present data shows a type 1 isotherm, and the Langmuir model was thus used to estimate surface area, as done previously for other carbon-based solids ${ }^{45}$. Figure $2 b$ shows the Langmuir linearization of the isotherm, and the derived surface area estimate of $1210 \mathrm{~m}^{2} \cdot \mathrm{g}^{-1}$. Bradder et al., 2011 reported that surface acidity in graphite oxide (the bulk form of graphene oxide) plays an important role in the adsorption of cationic dyes such as MB, and that electrostatic attraction is the primary mechanism. 
When GO goes through a chemical reduction process, the negative charge density decreases, and this has been shown to enhance anionic dye adsorption and reduce cationic dye adsorption 47.
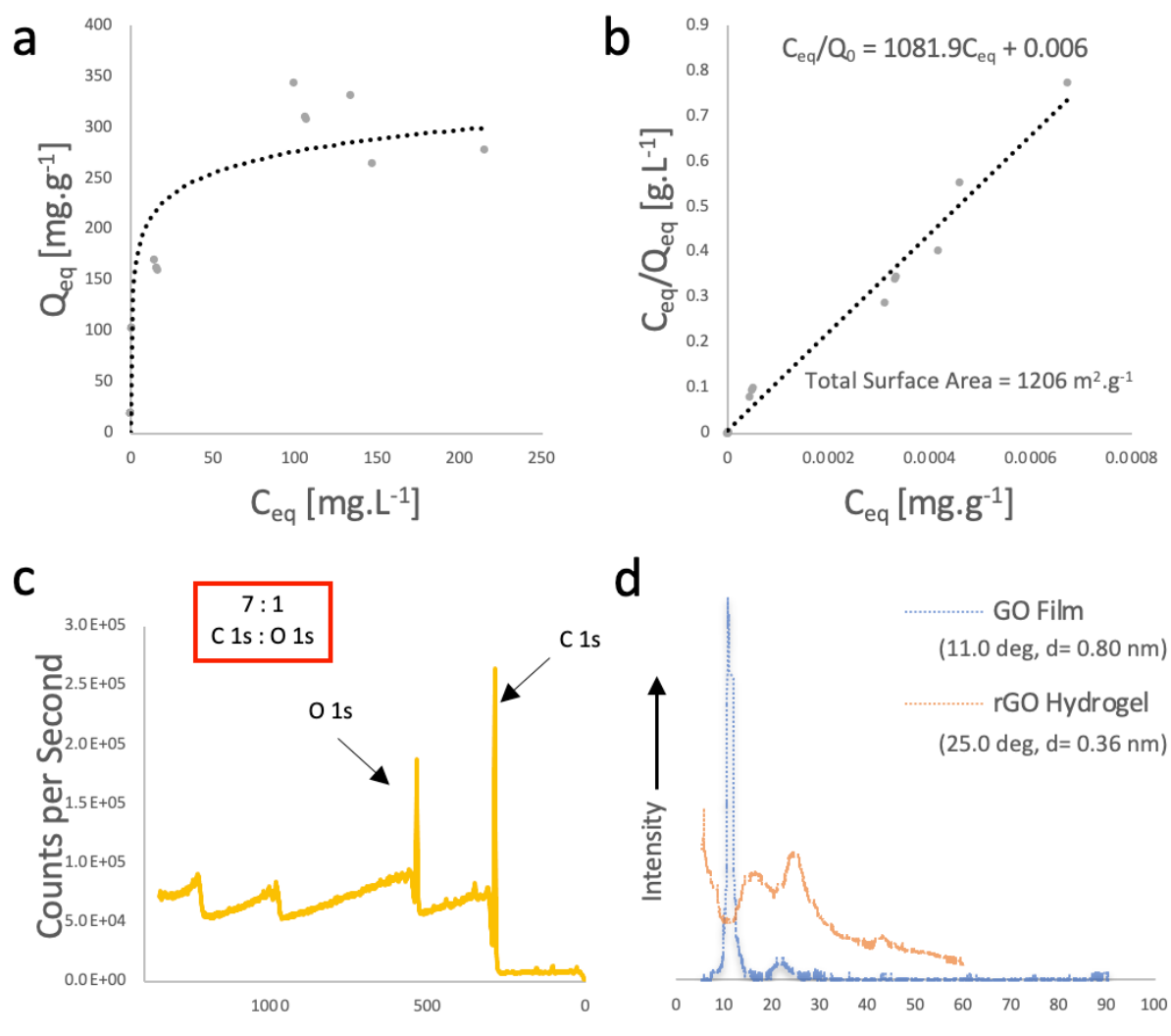

Binding Energy (eV)

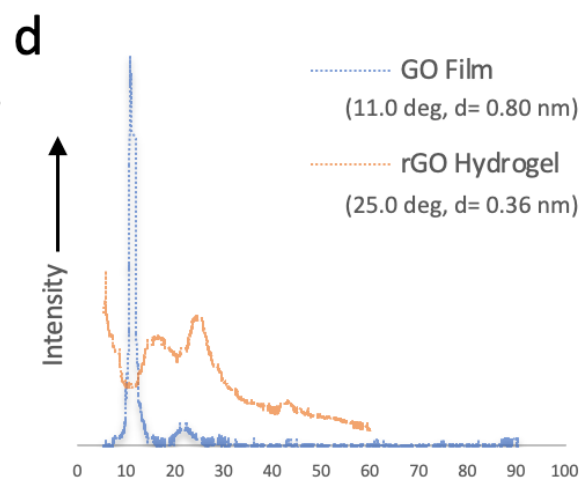

2 theta, degree

Figure 2: a) Methylene blue adsorption isotherm used for surface area determination; b) Linearization and fit to Langmuir isotherm. c) XPS spectra showed a change in the carbon oxygen ratio from $\sim 2: 1$ (original GO precursor [see SI]) to $\sim: 1$ in the hydrothermally treated monolith. d) XRD analysis also shows a change in the interlayer spacing from $0.80 \mathrm{~nm}(\mathrm{GO})$ to $0.36 \mathrm{~nm}$ ( $\mathrm{GO}-\mathrm{HG}$ ) which reflects the loss of oxygen functional groups between individual sheets.

Figure 2c shows XPS spectra revealing presence of both oxygen and carbon at $\sim 540$ and $\sim 295$ $\mathrm{eV}$ respectively, and an increase in the carbon:oxygen (C:O) atomic ratio from 2:1 (GO precursor) to $7: 1$ (final product). Figure SI 5 shows XPS data on stock graphene oxide used for comparison purposes. Figure $2 \mathrm{~d}$ shows comparative results between a thin $\mathrm{GO}$ film made from stock $\mathrm{GO}$ in suspension and the rGO monolith showing a shift in interlayer spacing from 0.80 to $0.36 \mathrm{~nm}$. Other hydrothermally prepared aerogels show similar patterns ${ }^{48}$ with loss of the $\sim 11^{\circ}$ peak and introduction of a broadened peak at $\sim 25^{\circ}$ associated with graphene layer pi-stacking. Our hydrothermal processing time was $10 \mathrm{hrs}$, while other hydrothermal studies report that GO 
deoxygenation reaches a maximum after about $6 \mathrm{hrs}{ }^{43,49}$ and further increases in C:O ratio are negligible.

\section{Surface charge modification through covalent grafting}

Short-chain PFAA are as environmentally persistent as their long-chained counterparts, and present a greater challenge for effective capture on adsorbents ${ }^{50,51}$. Carbon-based solids have been reported to be effective sorbents for long chain PFAS molecules but are less successful at capturing short chain PFAS $\left(<\mathrm{C}_{7}\right)^{52,53}$. This trend is attributed to the increased hydrophilicity of short-chain PFAS, and in turn, the decreased hydrophobic forces between shorter perfluorinated alkylchains and the carbon surface ${ }^{53}$. Electrostatic interactions are generally important forces in the adsorption of aliphatic anions to carbon basal planes ${ }^{54}$. These ideas suggest that varying adsorbent surface charge can be a useful tool to enhance the adsorption capacity of charged PFAS molecules, especially those with short chains where the hydrophobic driving force is weaker and electrostatic contributions are relatively more important.

Most PFAS are anionic, and most as-produced carbon or graphenic surfaces are negatively charged at near neutral $\mathrm{pH}$ due to acidic oxygen-containing functional groups, which arise either from the carbonization precursor or from chemisorption of atmospheric oxygen. The dual negative charges suggest that electrostatic repulsion may be limiting adsorption, especially for short-chain species where the weaker hydrophobic driving forces are more easily overcome. Figure $3 c$ shows that this specific graphene monolith, in its as-produced form, has a negative surface charge over the entire range of $\mathrm{pH}$ studied (3 to 11 ) and a large zeta potential of $-47 \mathrm{mV}$ near neutral $\mathrm{pH}$.

We hypothesized that the introduction of positively charged functional groups would decrease these repulsive forces and produce local net positive charges that favor the partitioning of shortchain anionic PFAS to the graphene surfaces. To introduce permanent positive surface charge, we sought a covalent grafting method compatible with graphene monoliths. First, the selected grafting method must be carried out in aqueous media to preserve hydrated state and monolith structure, and also must use reactive chemistry that avoids oxidative damage to the thin graphene walls. Many carbon functionalization protocols begin with $-\mathrm{COOH}$ introduction through treatment in concentrated oxidizing acids (e.g. $\mathrm{HNO}_{3}$ ), but these routes can easily damage or even destroy (mineralize) thin-walled carbon structures ${ }^{55,56}$. 


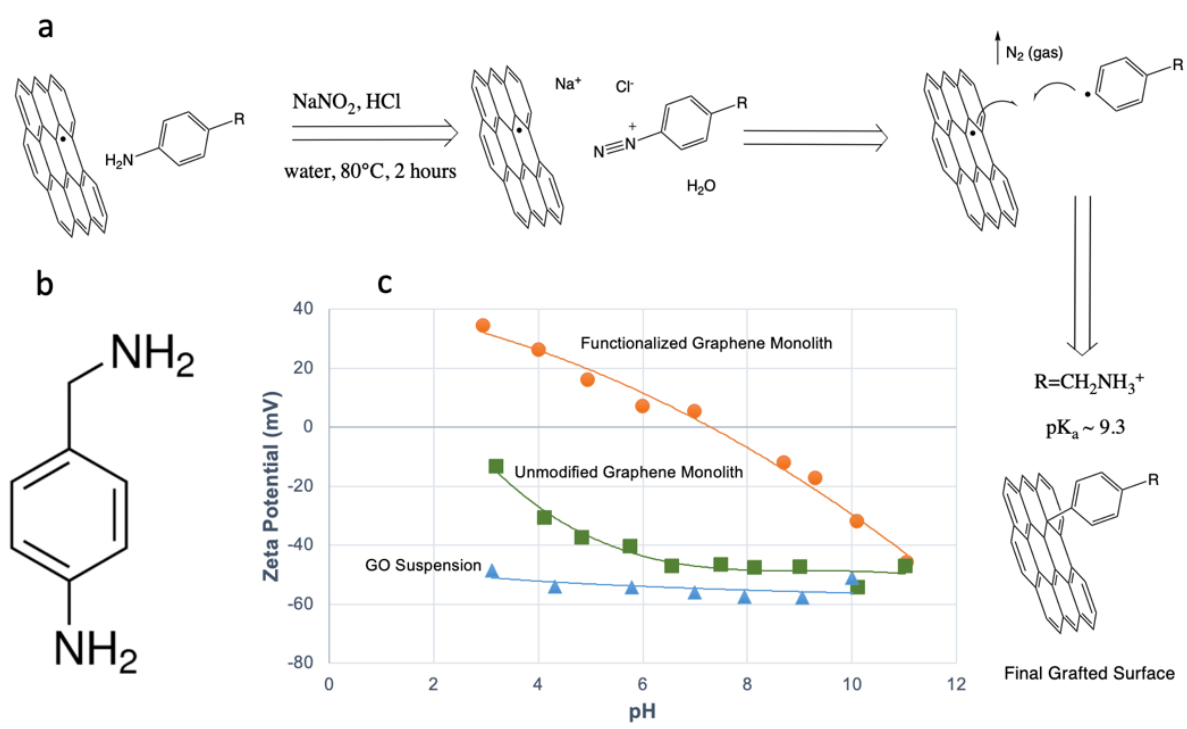

Figure 3. Covalent modification of graphene monoliths using diazonium chemistry: mechanisms and surface charge effects. a) Diazonium functionalization mechanism, where - $R$ is a general, nonspecified functional group to be introduced (Kariuki \& McDermott, 1999; Liu et al., 2014) b) Structure of 4-ABA used as a new precursor to introduce positive charge to the graphene surfaces up to neutral $\mathrm{pH}$ c) Measurement of $\mathrm{pH}$-dependent zeta potentials for the GO precursor suspension (blue), unmodified graphene monolith (green), and ABAfunctionalized graphene monolith (orange).

A mild, non-oxidizing, aqueous, and flexible class of methods for covalent grafting onto carbonbased surfaces is based on in situ generation of diazonium salts ${ }^{38,40}$. Figure $3 a$ illustrates this mechanism for the general case where "R" represents the target functional group to be introduced when the diazonium salt intermediate attaches to carbon surfaces through a covalent-aryl bond 39. A chosen aniline reagent containing " $R$ " in a mildly acidic aqueous solution at $80^{\circ} \mathrm{C}$ first reacts with a nitrosonium $\left(\mathrm{NO}^{+}\right)$ion, which is formed via the double protonation of $\mathrm{NO}_{2}^{-}$and the loss of a water molecule. The aniline nitrogen subsequently forms an $\mathrm{N}-\mathrm{N}$ bond with $\mathrm{NO}^{+}$, which then undergoes two successive protonations and an elimination of a water molecule, allowing for the formation of a nitrogen triple bond and a diazonium salt intermediate ${ }^{57}$. A free electron transfer from the rGO surface to the diazonium group liberates $\mathrm{N}_{2}$ gas and results in a reactive aryl radical 58. The radical then bonds with a free electron and couples to the carbon surface, with the entire process occurring in a single aqueous step ${ }^{59}$.

We sought a specific method within this class (an $\mathrm{R}$ group) to produce a stable positive charge up to $\mathrm{pH} 8.3$ for applications in natural waters ${ }^{60}$. Alkyl amines are suitable, having pKa values commonly >9. In general, amine-functionalized sorbents have shown great promise in the capture and remediation of aqueous PFAS, especially porous sorbents that retain hydrophobic segments in addition to electrostatic functionalities ${ }^{61}$. The simplest alkylamine derivative of aniline is 4aminobenzylamine (4-ABA) and was chosen here as the precursor for the diazonium reaction 
(Fig. 3b). The final grafted group has an outward-facing benzylamine structure with its conjugate acid having a pKa of $9.34{ }^{62}$.* Note that previous studies have used 4-ABA in surface functionalization, but using amine coupling chemistry rather than diazonium chemistry, and in this configuration the grafting site is reported to be the alkylamine, leaving the arylamine (aniline moiety) as the functional group presented to the environment, and this route would not introduce positive surface charge at neutral $\mathrm{pH}^{63,64}$.

Figure $3 c$ shows that 4-ABA functionalization dramatically alters the surface charge states of the graphene monolith. Across a wide $\mathrm{pH}$ range $(3-10)$, the functionalized monolith has a much greater zeta potential than the unmodified monolith, and a net positive charge is observed from $\mathrm{pH} 3$ - 7.5. At about $\mathrm{pH} 11$, the zeta potentials of original and functionalized monoliths become equal. This can be attributed to the loss of positive charge due to deprotonation of the benzylamine functional groups between $\mathrm{pH} 7$ and 11, which is consistent with the pKa of the conjugate acid of benzylamine (9.34). To our knowledge, this is the first time net positive charge has been introduced to a carbon surface through mild diazonium chemistry via a nonelectrochemical route. This one-pot diazonium functionalization mechanism represents a simple, novel way to introduce positive charge to the surfaces of the rGO monoliths under our desired pH conditions.

\footnotetext{
* Note that the symmetric para-phenylenediamine is a widely used additive, and was considered as an alternative, but it possesses an aromatic amine with a pKa value of only $6.2{ }^{65}$. The final grafted group would have the aniline structure with the aromatic amine facing outward (aniline conjugate acid $\mathrm{pKa}=4.59$ ) and would not exist in the majority protonated, positively charged state under most environmental $\mathrm{pH}$ values ${ }^{66}$.
} 


\section{PFAS adsorption and partition coefficients}

Time-dependent adsorption experiments over 48 hours were carried out on 16 PFAS species to establish the exposure times needed for equilibration (Table SI 5, Figure SI 1). Adsorbed amounts increase steadily over $8 \mathrm{hrs}$ and reach an apparent plateau from 12 - $48 \mathrm{hrs}$. This result confirms our initial hypothesis that the highly porous monolith structure would permit rapid equilibration, and further experiments were carried out at the 24 time point and the results analyzed using equilibrium-based models (isotherms and partition coefficients). Adsorbed amounts at equilibrium range from about 1 to $35 \mathrm{ng}$ PFAS / sampler (Table SI 5). This is a maximum mass loading of 35 $\mathrm{ng} / 11.2 \mathrm{mg} \sim 3.10^{-6}$. On a surface area basis, using $1210 \mathrm{~m}^{2} . \mathrm{g}^{-1}$ for the device, and typical molecular sizes and densities gives a PFAS fractional area coverage on the order of $10^{-6}$. Such extremely low surface coverage values reflect the ultra-dilute PFAS concentrations in solution that the samplers are in equilibrium with. Note that at high solute concentrations (see methylene blue data in Fig. 2a and b) these high-area solids can adsorb up to $30 \mathrm{wt}-\%$ organic adsorbates. For PFAS, both the mass-based and area-based fractional loadings are very small - of order 1 ppm, which strongly suggests that the partition coefficient approach is appropriate for quantifying performance.

Partition coefficients ( $K_{\text {sw }}$, Eq. 1) were therefore determined for 20 individual PFAS. The adsorbed amounts of individual PFAS on the pristine graphene monolith at equilibrium were calculated in ng. $\mathrm{g}^{-1}$, where the dry sampler mass was used for normalization (Table SI 8). Derived concentrations were corrected for the loses during the extraction by using mass labeled PFAS analogs (Table SI 1). The calculated log $\mathrm{K}_{\mathrm{sw}}$ values for targeted PFAS are shown Figure 4 and generally increase with increasing chain-length. In particular, log $K_{s w}$ values increase linearly with increasing chain length for perfluorinated sulfonic acids (PFSA, red) as well as for middle and long chain perfluorinated carboxylic acids (PFCA, light blue). C8 sulfonamide (FOSA, green) as well as its methyl- and ethyl- derivatives are strongly adsorbed to the graphene monolith in comparison with their PFCA and PFSA counterparts due to their hydrophobic characters ${ }^{42}$. Short chain PFAAs (dark blue) and short chain perfluoroalkane-ethers (HFPO-DA, yellow) do not significantly concentrate at the sampler $\left(\mathrm{K}_{\mathrm{SW}} \sim 1\right)$ relative to dissolved concentrations. This chain-

length trend is also seen in activated carbon adsorption ${ }^{50}$, and is believed to reflect the reduction in hydrophobic (attractive) forces with decreasing chain length, coupled in this case with the electrostatic repulsion between sorbent and adsorbate. 


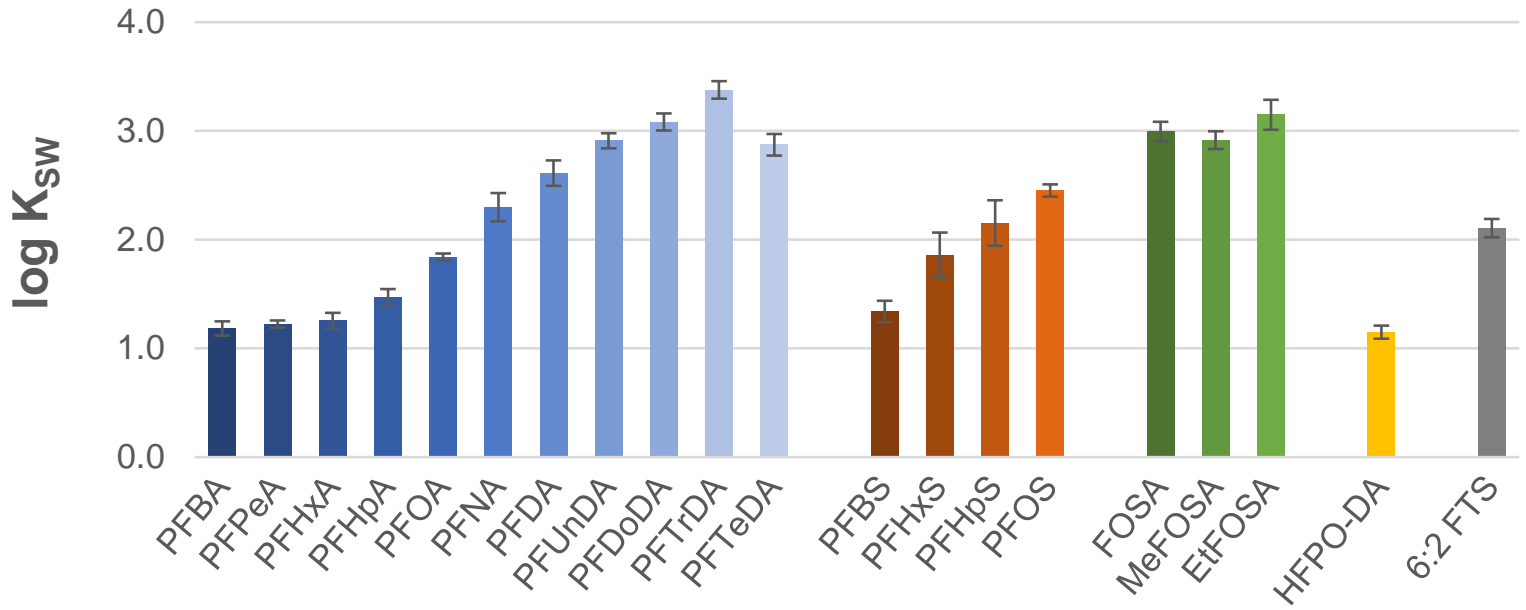

Figure 4 Partition coefficient measurements: Pristine (unmodified) graphene hydrogel sampler water partition coefficient (Ksw) for perfluoroalkyl carboxylic acids (PFCA, blue), perfluoroalkyl sulfonates (PFSA, red), perfluoroalkyl sulfonamides (green), HFPO-DA (yellow) and 6:2 FTS (grey).

\section{Adsorption isotherms}

Graphene monoliths (in triplicates) were exposed to a mixture of PFAS at 3 different concentrations (5, 50 and $500 \mathrm{ng} \cdot \mathrm{mL}^{-1}$ in $500 \mathrm{~mL}$ ) and adsorption isotherms for individual substances were derived. The data were fit to the Freundlich isotherm (Eq. 2) and the distribution coefficient $\left(K_{D}\right)$ for individual PFAS was calculated from its linearized form (Eq. 3)

Freundlich model of adsorption isotherm (Eq. 2) and its linear expression (Eq. 3):

$$
\begin{array}{rlrl}
q & =K_{D} \cdot C^{\frac{1}{n}} & E q .2 \\
\log q & =\frac{1}{n} \log C+\log K_{D} & E q .3
\end{array}
$$

Here $q$ is the amount of adsorption (moles adsorbate per mass unit of adsorbent), $K_{D}$ is distribution coefficient, $C$ is equilibrium concentration and $n$ is correction or nonlinearity factor (when $n=1$ then $\mathrm{K}_{\mathrm{D}}$ becomes the partition coefficient)

The regression coefficients and Pearson correlation coefficient for Freundlich isotherm model are presented in Table 1. Given the R-values (0.93-1.0), the Freundlich model describes the PFAS adsorption onto the graphene monolith well. For selected PFAS (PFDoDA, PFTrDA, PFTeDA, MeFOSA and EtFOSA) the factor correcting for nonlinearity $(n)$ exceeded 1 . This is most often attributed to adsorbate-adsorbate interactions ${ }^{67}$, creation of PFAS micelle at a critical concentration on the surfaces ${ }^{68}$ or losses due to PFAS irreversible sorption (potentially causing an underestimation of dissolved concentrations). Given that for most PFAS n was not significantly 
different from 1, the linear model ( $\mathrm{n}$ factor $=1$ ) was fitted to the data and the good agreement between the measured data and the model was achieved (Pearson coefficient in Table 1).

Table 1 Adsorption isotherms: Regression coefficients for Freundlich and linear models of adsorption isotherms $\left(1 / n, \log K_{D}\right)$ and Pearson correlation coefficient ( $r$ )

\begin{tabular}{lccccc}
\hline & \multicolumn{2}{c}{ Freundlich model } & \multicolumn{2}{c}{ Linear model } \\
\hline Compound & $\mathbf{1} / \mathbf{n}\left( \pm \mathbf{C l}^{\star}\right)$ & Log K & $\mathbf{R}$ & Log K & $\mathbf{R}$ \\
\hline PFBA & $0.99( \pm 0.36)$ & 1.20 & 1.00 & $1.19 \pm 0.05$ & 1.00 \\
PFPeA & $1.02( \pm 0.16)$ & 1.19 & 1.00 & $1.24 \pm 0.04$ & 1.00 \\
PFHxA & $1.00( \pm 0.66)$ & 1.28 & 1.00 & $1.27 \pm 0.04$ & 0.99 \\
PFHpA & $0.97( \pm 0.48)$ & 1.51 & 1.00 & $1.46 \pm 0.05$ & 0.99 \\
PFOA & $0.94( \pm 0.68)$ & 1.93 & 1.00 & $1.80 \pm 0.04$ & 0.99 \\
PFNA & $1.02( \pm 0.48)$ & 2.29 & 1.00 & $2.34 \pm 0.09$ & 1.00 \\
PFDA & $1.09( \pm 0.42)$ & 2.50 & 1.00 & $2.69 \pm 0.08$ & 0.99 \\
PFUnDA & $1.01( \pm 2.55)$ & 3.01 & 0.98 & $3.04 \pm 0.14$ & 0.98 \\
PFDoDA & $0.67( \pm 0.61)$ & 3.42 & 1.00 & $2.93 \pm 0.11$ & 0.97 \\
PFTrDA & $0.53( \pm 0.21)$ & 3.62 & 1.00 & $3.07 \pm 0.11$ & 0.98 \\
PFTeDA & $0.64( \pm 1.38)$ & 3.03 & 0.99 & $2.58 \pm 0.11$ & 0.99 \\
& & & & & \\
PFBS & $1.11( \pm 0.90)$ & 1.26 & 1.00 & $1.47 \pm 0.07$ & 1.00 \\
PFHxS & $0.96( \pm 0.90)$ & 1.92 & 1.00 & $1.86 \pm 0.08$ & 0.99 \\
PFHpS & $0.92( \pm 1.58)$ & 2.17 & 1.00 & $2.14 \pm 0.08$ & 0.99 \\
PFOS & $1.12( \pm 3.13)$ & 2.46 & 0.99 & $2.70 \pm 0.16$ & 0.99 \\
& & & & & \\
FOSA & $1.01( \pm 0.92)$ & 2.96 & 1.00 & $2.98 \pm 0.11$ & 0.99 \\
MeFOSA & $0.59( \pm 0.25)$ & 3.14 & 1.00 & $2.66 \pm 0.16$ & 0.98 \\
EtFOSA & $0.53( \pm 1.92)$ & 3.37 & 0.93 & $3.01 \pm 0.18$ & 0.82 \\
HFPO-DA & $1.00( \pm 0.62)$ & 1.13 & 1.00 & $1.13 \pm 0.05$ & 0.99 \\
6:2 FTS & $0.95( \pm 1.08)$ & 2.20 & 1.00 & $2.09 \pm 0.05$ & 0.98 \\
\hline & ${ }^{*} \mathrm{Cl}-95 \%$ confidence interval & & &
\end{tabular}

Performance of surface-modified graphene monoliths

Partition coefficients for PFAS onto graphene monoliths in the pristine and surface-modified forms were calculated and compared for 23 individual compounds. In each case, $11.2 \mathrm{mg}$ of each sampler (in replicates) were exposed to $100 \mathrm{~mL}$ of PFAS solution $\left(5 \mathrm{ng} \cdot \mathrm{mL}^{-1}\right)$ give that the previous results implied mostly linear sorption isotherms. We derived the $\mathrm{K}_{\mathrm{sw}}$ for PFAS compounds (Figure 5) and divided them into four subgroups based on their functional headgroups (Table 2). 


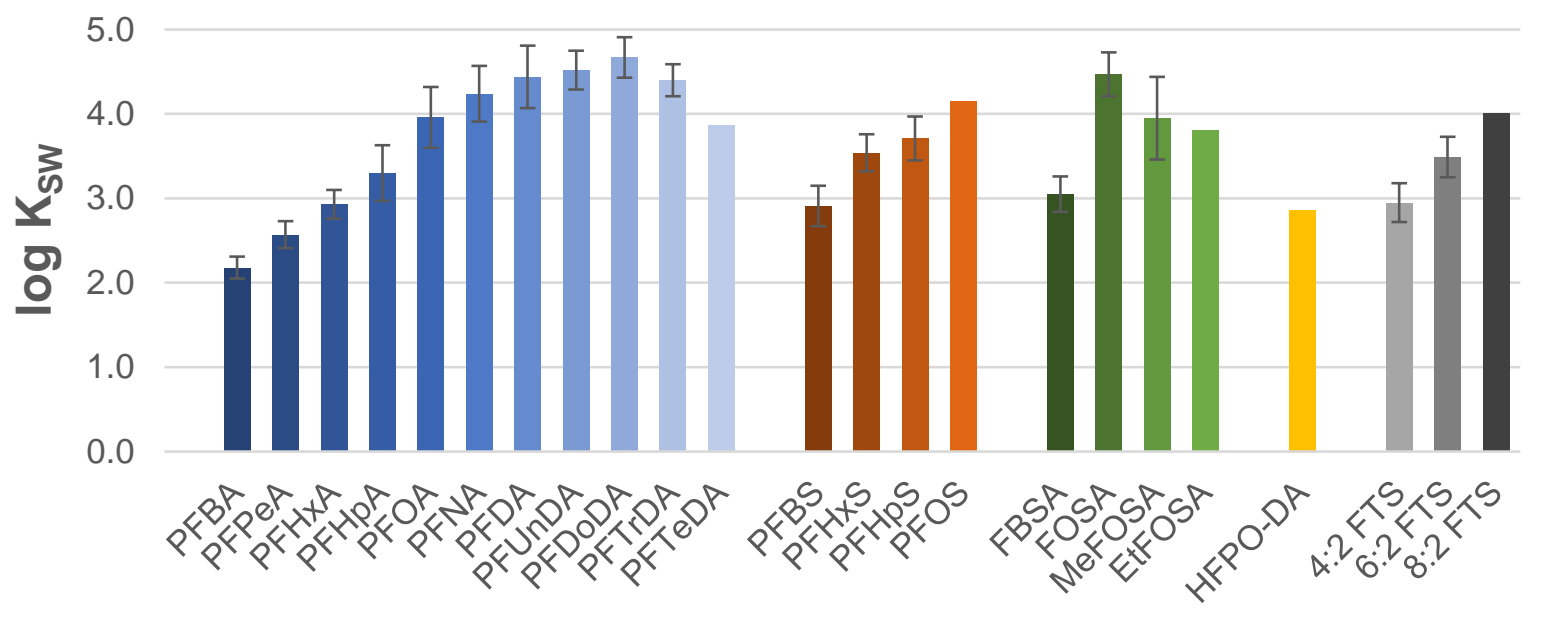

Figure 5 Partition coefficient: Surface-modified graphene hydrogel sampler water partition coefficient (Ksw) for perfluoroalkyl carboxylic acids (PFCA, blue), perfluoroalkyl sulfonates (PFSA, red), perfluoroalkyl sulfonamides (green), HFPO-DA (yellow) and FTSs (grey). 
Table 2 PFAS partition coefficients (log Ksw) for pristine and modified graphene monolith sampler: PFAS subgroups, 1 -perfluorinated carboxylic acids (PFCA, -COOH), 2 - perfluorinated sulfonic acids ( $P F S A$, - $\mathrm{SO}_{3} \mathrm{H}$ ), 3perfluorinated sulfonamides (- $\mathrm{SO}_{2} \mathrm{~N}=$ ), and 4 - fluorotelomer sulfonic acids ( $\mathrm{FTS},-\mathrm{SO}_{3} \mathrm{H}$ )

\begin{tabular}{|c|c|c|c|c|c|c|}
\hline & & & & & $\log K_{s w}$ & \\
\hline $\begin{array}{l}\text { Functional } \\
\text { group }\end{array}$ & Fluorination $^{1}$ & $\mathrm{n}\left(\mathrm{CF}_{2}\right)$ & Compound & $\begin{array}{l}\text { pristine } \\
\text { GM }^{*}\end{array}$ & $\begin{array}{c}\text { surface- } \\
\text { modified } \\
\text { GM }^{\# \#}\end{array}$ & $\otimes^{2}$ \\
\hline \multirow{11}{*}{$\begin{array}{c}1 \\
-\mathrm{COOH}\end{array}$} & \multirow{11}{*}{ Per- } & 3 & PFBA & $1.31 \pm 0.17$ & $2.18 \pm 0.07$ & $0.87^{*}$ \\
\hline & & 4 & PFPeA & $1.52 \pm 0.09$ & $2.57 \pm 0.13$ & $1.05^{\star *}$ \\
\hline & & 5 & PFHxA & $1.66 \pm 0.01$ & $2.93 \pm 0.16$ & $1.27^{* *}$ \\
\hline & & 6 & PFHpA & $1.93 \pm 0.06$ & $3.30 \pm 0.17$ & $1.37^{\star *}$ \\
\hline & & 7 & PFOA & $2.33 \pm 0.04$ & $3.96 \pm 0.33$ & $1.63^{\star *}$ \\
\hline & & 8 & PFNA & $2.62 \pm 0.06$ & $4.24 \pm 0.36$ & $1.62^{\star \star}$ \\
\hline & & 9 & PFDA & $2.63 \pm 0.01$ & $4.44 \pm 0.33$ & $1.81^{* *}$ \\
\hline & & 10 & PFUnDA & $3.03 \pm 0.04$ & $4.52 \pm 0.37$ & $1.49^{* *}$ \\
\hline & & 11 & PFDoDA & $3.43 \pm 0.12$ & $4.67 \pm 0.23$ & $1.24^{* *}$ \\
\hline & & 12 & PFTrDA & $3.90 \pm 0.19$ & $4.40 \pm 0.24$ & 0.50 \\
\hline & & 13 & PFTeDA & $4.37 \pm 0.19$ & $3.87 \pm 0.19$ & -0.50 \\
\hline \multirow{4}{*}{$\begin{array}{c}2 \\
-\mathrm{SO}_{3} \mathrm{H}\end{array}$} & \multirow{4}{*}{ Per- } & 4 & PFBS & $1.73 \pm 0.05$ & $2.91 \pm 0.17$ & $1.18^{* *}$ \\
\hline & & 6 & PFHxS & $2.29 \pm 0.03$ & $3.54 \pm 0.24$ & $1.25^{\star \star}$ \\
\hline & & 7 & PFHpS & $2.48 \pm 0.03$ & $3.71 \pm 0.22$ & $1.23^{\star \star}$ \\
\hline & & 8 & PFOS & $2.82 \pm 0.06$ & $4.15 \pm 0.26$ & $1.33^{* \star}$ \\
\hline \multirow{4}{*}{$\begin{array}{c}3 \\
-\mathrm{SO}_{2} \mathrm{~N}=\end{array}$} & \multirow{4}{*}{ Per- } & 4 & FBSA & $1.78 \pm 0.11$ & $3.05 \pm 0.17$ & $1.27^{*}$ \\
\hline & & 8 & FOSA & $3.78 \pm 0.06$ & $4.47 \pm 0.21$ & $0.69^{*}$ \\
\hline & & 8 & MeFOSA & $3.80 \pm 0.18$ & $3.95 \pm 0.26$ & 0.15 \\
\hline & & 8 & EtFOSA & $4.01 \pm 0.11$ & $3.81 \pm 0.49$ & -0.20 \\
\hline \multirow{4}{*}{$\begin{array}{c}4 \\
-\mathrm{SO}_{3} \mathrm{H}\end{array}$} & \multirow{4}{*}{ Poly- } & 5 & HFPO-DA & $1.68 \pm 0.03$ & $2.86 \pm 0.15$ & $1.18^{\star *}$ \\
\hline & & 4 & 4:2 FTS & $1.65 \pm 0.04$ & $2.95 \pm 0.18$ & $1.30^{* \star}$ \\
\hline & & 6 & 6:2 FTS & $2.24 \pm 0.12$ & $3.49 \pm 0.23$ & $1.25^{\star *}$ \\
\hline & & 8 & $8: 2$ FTS & $2.83 \pm 0.11$ & $4.01 \pm 0.24$ & $1.18^{\star \star}$ \\
\hline
\end{tabular}

1 per-fluorinated and poly-fluorinated carbon chains

2 GM and modified GM log $\mathrm{K}_{\text {sw }}$ delta $(\otimes)$, significance $\mathrm{p}$-value ${ }^{* *}<0.005,{ }^{*}<0.05$ number of replicates $n=\# 2, \# 5$

Given the difference between the PFAS partitioning to pristine and surface-modified graphene monolith, a significant improvement $(p<0.005)$ in partitioning towards modified GM was observed 
for all PFAS with log $\mathrm{K}_{\mathrm{Sw}}$ (pristine $\mathrm{GM}$ ) <3, including short and middle chain perfluorinated PFAA (group 1 and 2), perfluorinated ether (HPFO-DA) and polyfluorinated sulfonates (group 4).

Focusing only on the fluorinated carbons, a linear increase of log $\mathrm{K}_{\text {sw }}$ within each PFAS subgroup can be observed, both for fully (per)fluorinated PFAS (group 1, 2, and 3) and partially (poly)fluorinated FTS (group 4 in Table 2). The average increase from the pristine to the modified graphene monolith was 1.2 log units, or 30-times. The only exception was FOSA, for which partition coefficients were higher than expected based on chain length. In contrast to PFAAs, the sulfonamides (FOSA and alkyl-FOSA) are neutral (uncharged) molecules, which likely explains the smaller enhancement factors and $\otimes$ values (delta) achieved by introduction of positive charge relative to the PFAAs with their anionic head groups. Additionally, on the surface modified graphene monoliths the very long chain PFAAs $\left(\mathrm{CF}_{2} \geq 11\right)$ have lower partition coefficients than shorter PFAAs, which may be caused by the loss of hydrophobic, non-polar (carbon-centered) sites or by lower desorption efficiency during extraction.

When comparing the increase of $\delta \log \mathrm{K}_{\mathrm{sw}}$, either for the pristine or modified graphene monolith samplers, we found them largely consistent (between 0.26 and 0.36 unit increase for every additional $\mathrm{CF}_{2}$ - moiety) and can be used for predicting of environmental behavior ${ }^{69}$. This suggests (together with previous findings) that the perfluorinated chain is dominating the PFAS-graphene partitioning for longer chain lengths. However, the strong increase from pristine to surfacemodified monoliths also shows that electrostatic interactions increase partitioning by over one order of magnitude (average of 1.2 log units); or in other words the addition of a positive charge increases sorption as much as 4-5 $\mathrm{CF}_{2}$ - moieties. Knowing the respective roles of increasing $\mathrm{CF}_{2^{-}}$ moieties versus electrostatic interactions may be used to predict log $\mathrm{K}_{\mathrm{sw}}$ for unknown PFAS congeners detected on GM passive samplers.

\section{First proof-of-principle field demonstration of graphene monoliths as PFAS passive samplers}

Concentrations of PFAS in water (ng. $\mathrm{L}^{-1}$ ) at all locations derived from the graphene monolith results (Figure 6b, Table SI 9) were compared with PFAS concentrations (ng. $\mathrm{L}^{-1}$ ) obtained from grab water samples analysis (Figure 6a, Table SI 10). The PFAS composition was the same in both passive samplers and water grabs at all three locations, with elevated PFAS concentration present at the Site B. At this site, all legacy PFAS constituents (PFNA, PFOA, PFOS and 6:2 FTS) were captured by graphene monoliths as well as additional PFAS found in water at lower $\left(<5\right.$ ng. $\left.\mathrm{L}^{-1}\right)$ concentrations (PFDA, PFUnDA and PFTrDA). Overall, the derived water concentrations from graphene monolith sampler were 4-fold lower than the data obtained from analysis of the water with the factor consistent for all compounds at all locations. Overall, the field 
deployments demonstrate that the passive samplers can be successfully used to measure PFAS in the field. Future work will need to determine the reason for the differences between laboratoryand field-derived partitioning values for the graphene monoliths.

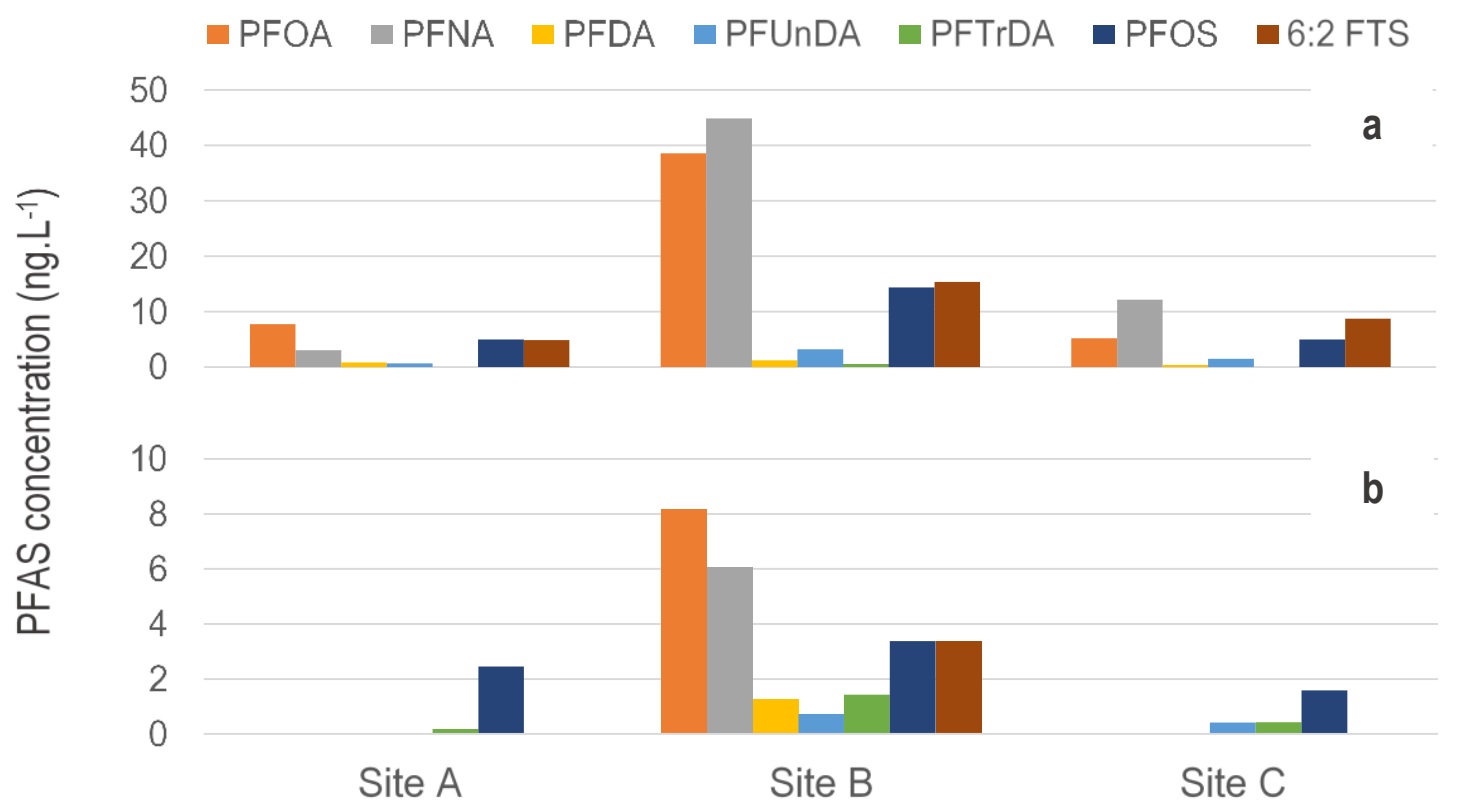

Figure 6 PFAs water concentration (ng. $\mathbf{L}^{-1}$ ) at 3 localities (Site A, Site B and Site C) in Delaware River (New Jersey, USA). The concentrations were derived from a) analysis of water grabs or b) analysis of graphene monoliths samplers.

To derive the detection limits (DL; ng/sampler) for a single graphene monolith and the overall method detection limits (MDL; ng/L) (Table SI 11) we used the LC-MS/MS detection limits determined as a 0.125 calibration curve point if no blank contamination was presented or the mean concentration in field blanks plus 3 times the standard deviation of the blank response (Table SI 9). The MDLs varies between sub nanogram per liter for the long chain PFCAs (C11C13), single ng per liter for the sulfonamides and most of the PFAA, and up to tens of ng per liter for the short chain PFCAs (C4-C6) and HFPO-DA (SI Table 11). The overall detection limits for a single graphene monolith are higher than recently tested PFAS integrative passive samplers such a POCIS ${ }^{70}$. This disadvantage can be easily overcome by creating samplers containing a larger GO mass or by co-deploying and pooling multiple graphene monoliths. A major advantage of the graphene monolith equilibrium sampler (in contrast to integrative passive samplers) is the short deployment time (days) along with the PFAS uptake independent on environmental conditions, such as water flow. 


\section{Conclusions}

Here we demonstrate that graphene oxide nanosheets can be hydrothermally assembled into porous graphene monoliths that act as miniature devices to concentrate PFAS from aqueous environments and enable their detection at trace concentrations. Devices fabricated with pristine graphene surfaces are effective at concentrating long-chain, but not short-chain PFAS in laboratory experiments. A new diazonium-based grafting chemistry based on aryl-amine precursors is shown to introduce positive charge to the graphene surfaces, which enables concentration of a broader range of PFAS species, including short-chain anionic compounds (carboxylic acids, sulfonic acids) that are challenging to capture by other techniques. Measured PFAS partition coefficients demonstrate significant correlations with chain length and molecular charge, which in the future may lead to generalized predictive methods for other PFAS species. Field deployment of the modified graphene monolith demonstrated its capability to quantitatively detect PFAS under environmentally relevant conditions (e.g., in a presence of co-contaminants and dissolve organic matter). This rapid equilibration of this aquatic passive sampling technology appears promising as an effective PFAS screening tool. Future work will focus on the formulation of the miniature monoliths as operator-friendly passive sampler devices, as well as validation of performance, recovery, and robustness in various water environments.

\section{Acknowledgments}

The authors acknowledge financial support from the National Institute of Environmental Health Sciences (NIEHS) through a KC Donnelly externship program (for Dr. Jitka Becanova); and the Superfund Research Program grants at the University of Rhode Island (grant P42-ES027706) and Brown University (grant P42-ES013660). The authors also acknowledge support from Strategic Environmental Research and Development Program (SERDP), Program Officer Andrea Leeson, contract ER20-1293. The authors acknowledge technical contributions from Grace Inman, Cintia Castilho, Muchun Liu, and Indrek Külaots at Brown University. 


\section{REFERENCES}

1 Glüge J, Scheringer M, Cousins IT, DeWitt JC, Goldenman G, Herzke D et al. An overview of the uses of per-and polyfluoroalkyl substances (PFAS). engrXiv 2020; 8: Web.

2 Posner S. Perfluorinated Compounds: Occurrence and Uses in Products. 2012.

3 Guelfo JL, Higgins CP. Subsurface Transport Potential of Perfluoroalkyl Acids at Aqueous FilmForming Foam (AFFF)-Impacted Sites. Environ Sci Technol 2013; 47: 4164-4171.

$4 \quad$ Place BJ, Field JA. Identification of Novel Fluorochemicals in Aqueous Film- Forming Foams (AFFF) Used by the US Military. Environ Sci Technol 2012; 46: 7120-7127.

5 Xiao X, Ulrich BA, Chen B, Higgins CP. Sorption of Poly- and Perfluoroalkyl Substances (PFASs) Relevant to Aqueous Film-Forming Foam (AFFF)-Impacted Groundwater by Biochars and Activated Carbon. Environ Sci Technol 2017; 51: 6342-6351.

6 Hu XDC, Andrews DQ, Lindstrom AB, Bruton TA, Schaider LA, Grandjean P et al. Detection of Poly- and Perfluoroalkyl Substances (PFASs) in US Drinking Water Linked to Industrial Sites, Military Fire Training Areas, and Wastewater Treatment Plants. Environ Sci Technol Lett 2016; 3: 344-350.

7 Arvaniti OS, Stasinakis AS. Review on the occurrence, fate and removal of perfluorinated compounds during wastewater treatment. Sci Total Environ 2015; 524-525: 81-92.

8 Hamid H, Li L. Role of wastewater treatment plant in environmental cycling of poly- and perfluoroalkyl substances. Ecocycles 2016; 2: 43-53.

9 Gallen C, Drage D, Eaglesham G, Grant S, Bowman M, Mueller JF. Australia-wide assessment of perfluoroalkyl substances (PFASs) in landfill leachates. J Hazard Mater 2017; 331: 132-141.

10 Hamid H, Li LY, Grace JR. Review of the fate and transformation of per- and polyfluoroalkyl substances (PFASs) in landfills. Environ Pollut 2018; 235: 74-84.

11 Lang JR, Allred BMK, Field JA, Levis JW, Barlaz MA. National Estimate of Per- and Polyfluoroalkyl Substance (PFAS) Release to U.S. Municipal Landfill Leachate. Environ Sci Technol 2017; 51: 2197-2205.

12 Corsini E, Luebke RW, Germolec DR, DeWitt JC. Perfluorinated compounds: Emerging POPs with potential immunotoxicity. Toxicol Lett 2014; 230: 263-270.

13 Dietert RR, Dewitt J. Developmental Immunotoxicity (DIT): the why, when, and how of DIT testing. Methods Mol Biol 2010; 598: 17-25.

14 Grandjean P, Andersen EW, Budtz-Jørgensen E, Nielsen F, Mølbak K, Weihe P et al. Serum Vaccine Antibody Concentrations in Children exposed to Perfluorinated compounds. JAMA 2012; 307: 391-397.

15 IARC. Some Chemicals Used as Solvents and in Polymer Manufacture IARC Monographs on the Evaluation of Carcinogenic Risks to Humans. Volume 110. Lyon, France, 2016.

EPA. Drinking Water Health Advisory for Perfluorooctane Sulfonate (PFOS). Washington, DC, 2016.

17 EPA. Drinking Water Health Advisory for Perfluorooctanoic Acid (PFOA). Washington, DC, 2016.

18 EPA. Human Health Toxicity Values for Hexafluoropropylene Oxide (HFPO) Dimer Acid and Its Ammonium Salt (CASRN 13252-13-6 and CASRN 62037-80-3) Also Known as "GenX Chemicals". Washingtoin DC, 2018.

UNEP. Stockholm Conventionon Persistent Organic Pollutants (POPs). 2009; : 64.

UNEP. Proposal to list pentadecafluorooctanoic acid (CAS No: 335-67-1, PFOA, perfluorooctanoic acid), its salts and PFOA-related compounds in Annexes A, B and/or $C$ to the Stockholm Convention on Persistent Organic Pollutants Stockholm Convention on Persistent O. 2015. 
21 Sunderland EM, Hu XC, Dassuncao C, Tokranov AK, Wagner CC, Allen JG. A review of the pathways of human exposure to poly- and perfluoroalkyl substances (PFASs) and present understanding of health effects. J Expo Sci Environ Epidemiol 2019; 29: 131-147.

22 EFSA EFSA. Risk to human health related to the presence of perfluoroalkyl substances in food. EFSA J 2020; 18: e06223.

Post GB. Recent US State and Federal Drinking Water Guidelines for Per- And Polyfluoroalkyl Substances (PFAS). Environ Toxicol Chem 2020; n/a. doi:10.1002/etc.4863.

Gobelius L, Persson C, Wiberg K, Ahrens L. Calibration and application of passive sampling for per- and polyfluoroalkyl substances in a drinking water treatment plant. $J$ Hazard Mater 2019; 362 : 230-237.

Kaserzon SL, Kennedy K, Hawker DW, Thompson J, Carter S, Roach AC et al. Development and calibration of a passive sampler for perfluorinated alkyl carboxylates and sulfonates in water. Environ Sci Technol 2012; 46: 4985-4993.

Kaserzon SL, Vermeirssen ELM, Hawker DW, Kennedy K, Bentley C, Thompson J et al. Passive sampling of perfluorinated chemicals in water: Flow rate effects on chemical uptake. Environ Pollut 2013; 177: 58-63.

Kaserzon SL, Hawker DW, Booij K, O’Brien DS, Kennedy K, Vermeirssen ELM et al. Passive sampling of perfluorinated chemicals in water: In-situ calibration. Environ Pollut 2014; 186: 98 103.

Li Y, Yang C, Bao Y, Ma X, Lu G, Li Y. Aquatic passice sampling of perfluorinated chemicals with polar organic chemical integrative sampler and environmental factors affecting sampling rate. Environ Sci Pollut Res 2016; 23: 16096-16103.

Wang L, Gong X, Wang R, Gan Z, Lu Y, Sun H. Application of an immobilized ionic liquid for the passive sampling of perfluorinated substances in water. J Chromatogr A 2017; 1515: 45-53.

Fauvelle V, Kaserzon SL, Montero N, Lissalde S, Allan IJ, Mills G et al. Dealing with Flow Effects on the Uptake of Polar Compounds by Passive Samplers. Environ Sci Technol 2017; 51: 25362537.

Guan D-X, Li Y-Q, Yu N-Y, Yu G-H, Wei S, Zhang H et al. In situ measurement of perfluoroalkyl substances in aquatic systems using diffusive gradients in thin-films technique. Water Res 2018; 144: $162-171$.

Urik J, Vrana B, Urík J, Vrana B. An improved design of a passive sampler for polar organic compounds based on diffusion in agarose hydrogel. Environ Sci Pollut Res 2019. doi:10.1007/s11356-019-04843-6.

Novoselov KS, Geim AK, Morozov S V, Jiang D, Zhang Y, Dubonos S V et al. Electric Field Effect in Atomically Thin Carbon Films. Science (80-) 2004; 306: 666 LP - 669.

Guo F, Creighton M, Chen Y, Hurt R, Külaots I. Porous structures in stacked, crumpled and pillared graphene-based 3D materials. Carbon N Y 2014; 66: 476-484.

Joshi RK, Carbone P, Wang FC, Kravets VG, Su Y, Grigorieva I V et al. Precise and Ultrafast Molecular Sieving Through Graphene Oxide Membranes. Science (80- ) 2014; 343: 752 LP - 754.

Spitz Steinberg R, Cruz M, Mahfouz NGA, Qiu Y, Hurt RH. Breathable Vapor Toxicant Barriers Based on Multilayer Graphene Oxide. ACS Nano 2017; 11: 5670-5679.

37 Liu M, Weston PJ, Hurt RH. Controlling nanochannel orientation and dimensions in graphenebased nanofluidic membranes. Nat Commun 2021; 12: 1-7.

38 Liu M, Duan Y, Wang Y, Zhao Y. Diazonium functionalization of graphene nanosheets and impact response of aniline modified graphene/bismaleimide nanocomposites. Mater Des 2014; 53: 466474. 

of single-walled carbon nanotube functionalization. Science (80- ) 2003; 301: 1519-1522.

40 Yan A, Xiao X, Külaots I, Sheldon BW, Hurt RH. Controlling water contact angle on carbon surfaces from $5^{\circ}$ to $167^{\circ}$. Carbon N. Y. 2006; 44: 3116-3120.

41 McCord JP, Strynar MJ, Washington JW, Bergman EL, Goodrow SM. Emerging Chlorinated Polyfluorinated Polyether Compounds Impacting the Waters of Southwestern New Jersey Identified by Use of Nontargeted Analysis. Environ Sci Technol Lett 2020; 7: 903-908.

Kim M, Li LY, Grace JR, Yue C. Selecting reliable physicochemical properties of perfluoroalkyl and polyfluoroalkyl substances (PFASs) based on molecular descriptors. Environ Pollut 2015; 196: 462-472.

Xu Y, Sheng K, Li C, Shi G. Self-assembled graphene hydrogel via a one-step hydrothermal process. ACS Nano 2010; 4: 4324-4330.

44 Zhang H, Han X, Yang Z, Zou J, Tang H. Enhanced Adsorption of Methylene Blue on Graphene Oxide by Tuning the Oxidation Degree of Graphene Oxide. J Nanomater Mol Nanotechnol 2013; s1. doi:10.4172/2324-8777.s1-003.

Itodo AU, Itodo HU, Gafar MK. Estimation of Specific Surface Area using Langmuir Isotherm Method. J App/ Sci Environ Manag 2010; 14: 141-145.

Bradder P, Ling SK, Wang S, Liu S. Dye adsorption on layered graphite oxide. J Chem Eng Data 2011; 56: 138-141.

47 Ramesha GK, Vijaya Kumara A, Muralidhara HB, Sampath S. Graphene and graphene oxide as effective adsorbents toward anionic and cationic dyes. J Colloid Interface Sci 2011; 361: 270-277.

Gao C, Dong Z, Hao X, Yao Y, Guo S. Preparation of Reduced Graphene Oxide Aerogel and Its Adsorption for $\mathrm{Pb}(\mathrm{II})$. ACS Omega 2020; 5: 9903-9911.

Hu K, Xie X, Szkopek T, Cerruti M. Understanding Hydrothermally Reduced Graphene Oxide Hydrogels: From Reaction Products to Hydrogel Properties. Chem Mater 2016; 28: 1756-1768.

Brendel S, Fetter É, Staude C, Vierke L, Biegel-Engler A. Short-chain perfluoroalkyl acids: environmental concerns and a regulatory strategy under REACH. Environ Sci Eur 2018; 30. doi:10.1186/s12302-018-0134-4.

51 Gagliano E, Sgroi M, Falciglia PP, Vagliasindi FGA, Roccaro P. Removal of poly- and perfluoroalkyl substances (PFAS) from water by adsorption: Role of PFAS chain length, effect of organic matter and challenges in adsorbent regeneration. Water Res 2020; 171: 115381.

McCleaf P, Englund S, Östlund A, Lindegren K, Wiberg K, Ahrens L. Removal efficiency of multiple poly- and perfluoroalkyl substances (PFASs) in drinking water using granular activated carbon (GAC) and anion exchange (AE) column tests. Water Res 2017; 120: 77-87.

Vo HNP, Ngo HH, Guo W, Hong Nguyen TM, Li J, Liang H et al. Poly-and perfluoroalkyl substances in water and wastewater: A comprehensive review from sources to remediation. $J$ Water Process Eng 2020; 36: 101393.

54 Radovic LR, Silva IF, Ume JI, Menéndez JA, Leon Y Leon CA, Scaroni AW. An experimental and theoretical study of the adsorption of aromatics possessing electron-withdrawing and electrondonating functional groups by chemically modified activated carbons. Carbon N Y 1997; 35: 13391348.

Hu H, Zhao B, Itkis ME, Haddon RC. Nitric Acid Purification of Single-Walled Carbon Nanotubes. J Phys Chem B 2003; 107: 13838-13842.

56 Rosca ID, Watari F, Uo M, Akasaka T. Oxidation of multiwalled carbon nanotubes by nitric acid. Carbon N Y 2005; 43: 3124-3131.

57 Schank K. Preperation of diazonium groups. In: The chemistry of diazonium and diazo groups. 
1978, pp 645-658.

58 Kasprzak A, Zuchowska A, Poplawska M. Functionalization of graphene: does the organic chemistry matter? Beilstein J Org Chem 2018; 14: 2018-2026.

59 Dyke CA, Tour JM. Unbundled and highly functionalized carbon nanotubes from aqueous reactions. Nano Lett 2003; 3: 1215-1218.

60 Marion GM, Millero FJ, Camões MF, Spitzer P, Feistel R, Chen CTA. PH of seawater. Mar Chem 2011; 126: 89-96.

61 Ateia M, Alsbaiee A, Karanfil T, Dichtel W. Efficient PFAS Removal by Amine-Functionalized Sorbents: Critical Review of the Current Literature. Environ Sci Technol Lett 2019; 6: 688-695.

62 Hall HK. Correlation of the Base Strengths of Amines. J Am Chem Soc 1957; 79: 5441-5444.

63 Jeong KM, Li Y, Yoo DG, Lee NK, Lee HG, Ando S et al. Effects of crosslinking agents on the physical properties of polyimide/amino-functionalized graphene oxide hybrid films. Polym Int 2018. doi:10.1002/pi.5555.

64 Lee D, Choi MC, Ha CS. Polynorbornene dicarboximide/amine functionalized graphene hybrids for potential oxygen barrier films. J Polym Sci Part A Polym Chem 2012; 50: 1611-1621.

65 Meyer A, Fischer K. Oxidative transformation processes and products of para-phenylenediamine (PPD) and para-toluenediamine (PTD)—a review. Environ Sci Eur 2015; 27. doi:10.1186/s12302015-0044-7.

66 Bryson A. The Effects of m-Substituents on the pKa Values of Anilines, and on the Stretching Frequencies of the N-H Bonds. J Am Chem Soc 1960; 82: 4858-4862.

67 Unuabonah El, Omorogie MO, Oladoja NA. Modeling in Adsorption: Fundamentals and Applications. 2019.

68 Costanza J, Arshadi M, Abriola LM, Pennell KD. Accumulation of PFOA and PFOS at the AirWater Interface. Environ Sci Technol Lett 2019; 6: 487-491.

69 Higgins CP, Luthy RG. Surfactants onto Sediment Materials : An a priori Approach for Perfluoroalkyl Surfactants and Linear Alkylbenzene Sulfonates. Environ Sci Technol 2007; 41: 3254-3261.

70 Lai FY, Rauert C, Gobelius L, Ahrens L. A critical review on passive sampling in air and water for per- and polyfluoroalkyl substances (PFASs). TrAC Trends Anal Chem 2019.

doi:10.1016/J.TRAC.2018.11.009. 